\title{
PERFIL SOROLÓGICO EM POTENCIAIS DOADORES DE ÓRGÃOS SÓLIDOS DE SANTA CATARINA NO PERÍODO DE 2001 A 2007
}

\author{
Serologic potential in solid organ donors in Santa Catarina from 2001 to 2007
}

\author{
Rosana Marquardt Baumel, Eleonora D’Orsi, Emil Kupek
}

\section{RESUMO}

Objetivo: O objetivo deste estudo foi analisar o perfil sorológico entre os potenciais doadores de órgãos sólidos no Estado de Santa Catarina no período de janeiro de 2001 a dezembro de 2007. Métodos: Foi conduzido estudo epidemiológico, transversal baseado em dados secundários. A população estudada foi composta por 1424 potenciais doadores de órgãos sólidos. O perfil sorológico das doenças da população do estudo foi realizado pelo Centro de Hematologia e Hemoterapia de Santa Catarina, HEMOSC e os dados sócio-econômicos foram captados pela Central de Notificação, Captação e Distribuição de Órgãos de Santa Catarina (CNCDO/SC). As variáveis analisadas neste estudo foram os resultados das sorologias segundo gênero, idade e ano do exame. Resultados: O estudo constitui-se de 1424 resultados dos testes sorológicos de potenciais doadores de órgãos sólidos, 551 (38,7\%) do gênero feminino e 873 (61,3\%), do gênero masculino. Os resultados foram: anti-HBc $=21,7 \%, \mathrm{HBsAg}=3,9 \%$, anti-HCV $=1,3 \%$, doença de Chagas $=0,3 \%$, sífilis $=0,9 \%$, $\mathrm{HTLV} \mathrm{I} / \mathrm{II}=0,3 \%$, HIV I/II $=0,5 \%$, toxo $\operatorname{IgG}=38,2 \%$, toxo $\operatorname{IgM}=2,3 \%$, CMV $\operatorname{IgG}=46,2 \%$ e $\mathrm{CMV} \operatorname{IgM}=2,6 \%$. A soroprevalência de CMV IgM foi maior nas mulheres ( $p<0,01)$. Os outros marcadores não apresentaram diferença estatisticamente significativa entre os gêneros. Conclusões: Houve maior prevalência do gênero masculino entre os potenciais doadores de órgãos e aumento na faixa etária acima de 60 anos. O marcador HBsAg foi maior em homens durante o período estudado. As soroprevalências de HCV, HIV I/II, HTLV I/II e doença de Chagas foram baixas, sem alterações ao longo do período estudado. As infecções por citomegalovírus e toxoplasmose mostraram-se elevadas, sendo a infecção aguda pouco comum. Citomegalovírus foi mais frequente nas mulheres e nos mais jovens.

Descritores: Hepatite B, Hepatite C, HIV, Vírus Linfotrópico de Células T Humanas Tipo 1, Doença de Chagas, Citomegalovírus, Sífilis, Toxoplasmose, Soroprevalência, Transplante de Órgãos.

\section{Instituição:}

Departamento de Saúde Pública da Universidade Federal de Santa Catarina, Campus Universitário Trindade - Florianópolis - Santa Catarina - Brasil

\section{Correspondência:}

Rosana Marquardt Baumel

Departamento de Saúde Pública, Universidade Federal de Santa Catarina, Campus Universitário Trindade, CEP 88040-900, Florianópolis/SC

Tel: (48) 3721-9000

E-mail: baumelrosana@hotmail.com

Recebido em: 30.09 .2010

Aceito em: 09.07.2011

\section{INTRODUÇÃO}

O desenvolvimento das técnicas de transplante de órgãos e sua aplicação como tratamento de doenças constitui-se em um dos mais bem sucedidos avanços mundiais na área da saúde. ${ }^{1}$ Com a melhoria das técnicas cirúrgicas e da medicação imunossupressora, os transplantes tornaram-se mais efetivos devido à menor taxa de rejeição. Houve uma evolução considerável em termos de variedade de órgãos transplantados e procedimentos realizados. ${ }^{2}$

No Brasil, há um desenvolvimento crescente nesse setor. Em 2005, foram realizados 2730 transplantes de órgãos e tecidos por um milhão de habitantes (pmp), o que representa um aumento de $18,3 \%$ com relação ao ano de 2003 (2342 pmp) e de 36,6\% com relação a ano de 2002 (2056 pmp). ${ }^{3}$

Esse crescimento no número de doações realizadas é consequência da conscientização da população brasileira, da atuação competente das equipes e instituições autorizadas pelo Sistema Nacional de Transplantes de Órgãos do Ministério da Saúde e da regulação desse sistema, fundamentada na legislação vigente. ${ }^{3} \mathrm{O}$ transplante pode prolongar e melhorar a qualidade de vida, sendo muitas vezes a única alternativa para a sobrevivência do paciente. ${ }^{3}$

Apesar do crescimento da quantidade de doações, a relação entre o número de doadores de órgãos e a demanda por transplantes não é satisfatória. Segundo Garcia et al, o grande problema enfrentado pelos pacientes que aguardam por um transplante é a diferença entre a demanda de pacientes que estão na fila e o número de órgãos disponibilizados. ${ }^{4}$ 
No Brasil, segundo o Sistema Nacional de Transplantes, a taxa de doadores efetivos por milhão de população em 2007 foi de 6,2 pmp e em 2006 foi de 6,0 - uma alta de 3,33\%. ${ }^{4}$ Em 2008 a média nacional foi de $7,2 \mathrm{pmp}^{5}$

Santa Catarina, com 16,7 doadores efetivos pmp, é destaque como o estado brasileiro que mais capta órgãos e tecidos para transplante. $\mathrm{O}$ número de doações foi crescente, porém a fila de espera de pessoas que necessitam de um órgão transplantado também aumentou. ${ }^{5} \mathrm{O}$ perfil sorológico dos potenciais doadores de órgãos obtidos por meio das taxas de prevalência dos marcadores das doenças é um importante passo na notificação de um potencial doador. ${ }^{6}$ A soropositividade para alguns marcadores não impede que a doação seja realizada e para outros pode determinar o fim do processo de transplante.

Contra indicações absolutas à doação de órgãos incluem infecção pelos vírus HIV I/II e HTLV I/II. A presença dos marcadores para hepatite $\mathrm{B}$ e $\mathrm{C}$ é contra indicações relativas à doação de órgãos. A transmissão viral certamente ocorre com o transplante de órgãos sólidos, mas os resultados pós-transplante são aceitáveis, não havendo aumento na mortalidade ou na morbidade dos receptores. ${ }^{8}$ Quanto às infecções por outros marcadores; não há contra indicação absoluta, porém as infecções através de órgãos sólidos podem determinar a diminuição do sucesso do procedimento. A profilaxia de rotina tem sido utilizada e reduz expressivamente a morbimortalidade associada à infecção por esses agentes. ${ }^{9}$

Em doações de órgãos, é obrigatória a realização de exames laboratoriais para identificação de doenças transmissíveis pelo sangue e outros tecidos ou órgãos. ${ }^{10}$ Os órgãos a serem doados não podem ser transplantados antes da triagem sorológica dos marcadores anti-HBc, HBsAg; anti-HCV, HIV I/II, HTLV I/II, citomegalovírus (CMV IgG e CMV IgM), sífilis, toxoplasmose (toxo IgG e toxo $\operatorname{IgM}$ ) e doença de Chagas. Os exames para a identificação dos marcadores das doenças devem ser feitos em amostras testadas, registrados na ANVISA (Agência Nacional de Vigilância Sanitária). O objetivo da vigilância sanitária na área de doação de órgãos é minimizar os riscos de contaminação póstransplantes, por meio da formulação e aplicação de mecanismos que visem maior proteção para a saúde dos doadores e receptores. ${ }^{4}$ $\mathrm{O}$ rastreamento sorológico tem se constituído em uma fonte rotineira de informações epidemiológicas para a estimativa das taxas de prevalência dos marcadores de doenças.

A relevância social e científica dessa pesquisa epidemiológica dá-se pela originalidade do tema, visto que até o presente momento, em Santa Catarina, não existem dados disponíveis do perfil sorológico dos marcadores de doenças transmissíveis nessa série temporal (2001-2007) em doadores de órgãos.

O objetivo deste estudo foi verificar o perfil sorológico dos marcadores de doenças na população de potenciais doadores de órgãos sólidos no Estado de Santa Catarina, no período de 2001 a 2007 segundo gênero e idade.

\section{MÉTODOS}

Foi conduzido um estudo epidemiológico, transversal baseado em dados secundários. A população investigada foi constituída por potenciais doadores de órgãos sólidos do Estado de Santa Catarina, cadastrados na $\mathrm{CNCDO} / \mathrm{SC}$ e que tiveram o sangue testado no HEMOSC, órgãos vinculados à Secretaria de Estado da Saúde de Santa Catarina.

Os dados das sorologias do banco de dados secundários do HEMOSC foram digitados no computador pela pesquisadora, no programa Epidata Entry ${ }^{11} \mathrm{e}$, após a digitação das sorologias, foram incluídos os dados da CNCDO/SC (idade e gênero).

Os dados coletados na $\mathrm{CNCDO} / \mathrm{SC}$ estavam anotados em livros

Figura 1: Métodos empregados na pesquisa sorológica pelo Centro de Hematologia e Hemoterapia de Santa Catarina (HEMOSC)

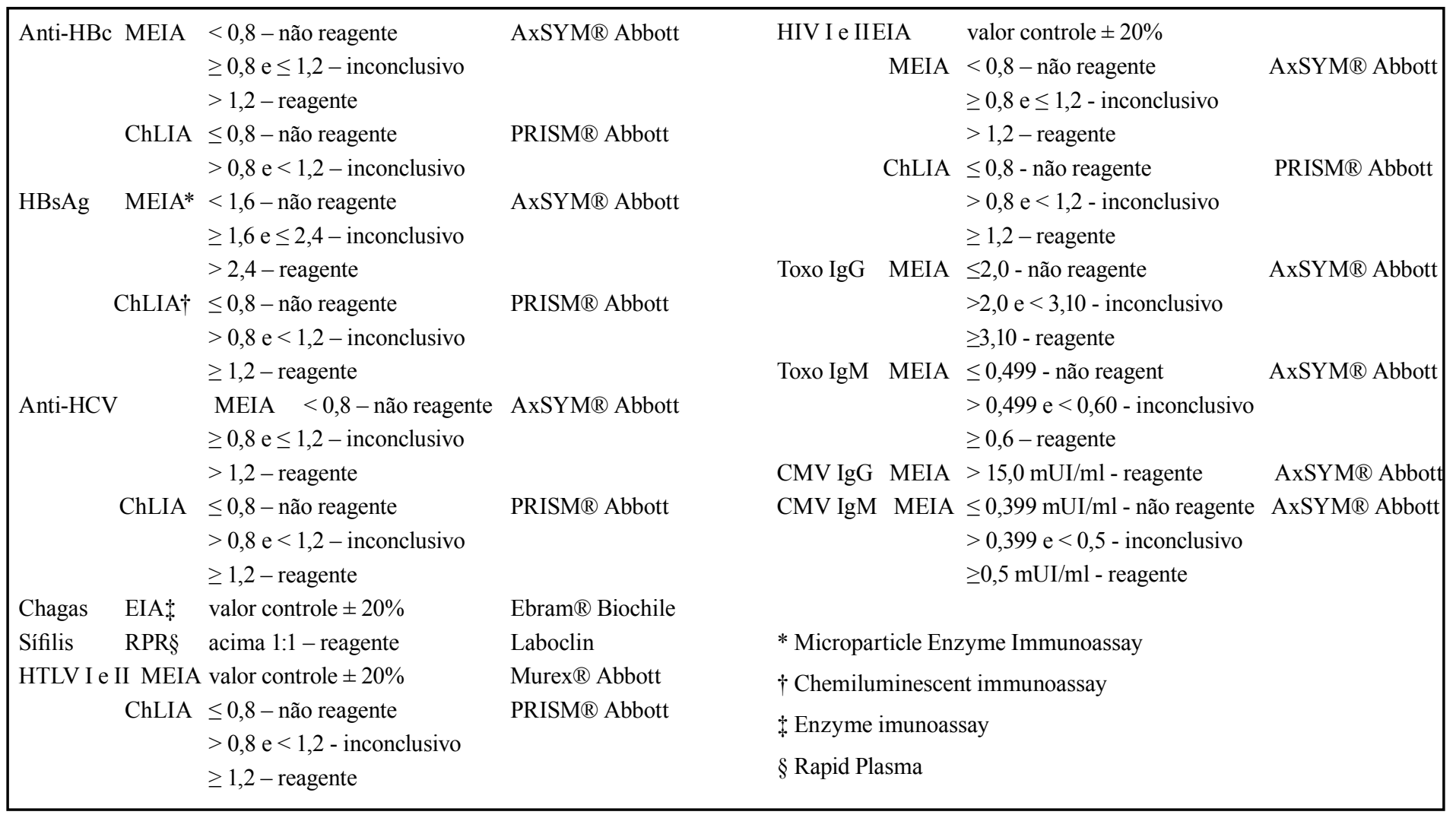


de notificações de candidatos à doação de forma sequencial, desde 2001 até 2007.

Somente a partir de 2006 a pesquisa dos marcadores sorológicos passou a ser padronizada, sendo todas as sorologias testadas no HEMOSC. Antes desse período os testes eram realizados nos municípios de origem do potencial doador.

Em relação à toxoplasmose, somente no final do ano de 2005 a sorologia para essa doença passou a ser rotina no processo de triagem ${ }^{6}$ e desta forma apenas os dados referentes a 2006 e 2007 representam o total de doadores testados neste trabalho.

As variáveis dependentes do estudo foram os resultados classificados em negativo, positivo, indeterminado e inconclusivo, das sorologias para: hepatite $\mathrm{B}$ (anti-HBc), antígeno de superfície (s) da hepatite B (HBsAg), anticorpo contra hepatite C, (anti-HCV) anticorpo contra doença de Chagas, anticorpo para sífilis, anticorpo contra o vírus T-Linfotrópico Humano (HTLVI/II); anticorpo vírus da imunodeficiência humana (HIVI/II); anticorpo contra toxoplasmose (toxo $\operatorname{IgG}$ e toxo $\operatorname{IgM}$ ), anticorpo contra antígeno do vírus citomegalovírus (CMV IgG e CMV IgM).

As variáveis independentes do estudo foram: a idade dos candidatos à doação de órgãos, sendo classificados em sete categorias (faixas etárias): menores de 10 anos, de 10-19, de 20-29, de 30-39, de 40-49, de 50-59 e de 60 anos e mais; o ano em que os potenciais doadores de órgãos foram submetidos à sorologia (2001-2007) e o gênero dos candidatos.

Os métodos empregados para cada sorologia dos potenciais doadores de órgãos sólidos no laboratório do HEMOSC seguem o mesmo padrão da triagem sorológica para doadores de sangue, conforme mostrado na Figura 1.

Para detectar a presença da reação antígeno-anticorpo, a técnica mais utilizada é a de imunoensaio de quimiluminescência (Chemiluminescent Immunoassay-ChLIA). A Prism ${ }^{\circledR}$ da Abbott é a plataforma onde são processadas as amostras. Essa técnica é utilizada para detectar os anticorpos: anti-HBc, anti-HCV, HTLV I e II, HIV I e II e o antígeno HBsAg.

Para a detecção dos marcadores para toxoplasmose (toxo-IgG e toxo $\operatorname{IgM}$ ) e citomegalovírus (CMV IgG e CMV IgM), a técnica é executada por imunoensaio enzimático de micropartículas (Microparticle Enzyme Immunoassay- MEIA), da empresa Abbott.

Para a triagem da sífilis é realizado o teste da Rapid Plasma ReaginRPR, uma variante do teste Veneral Disease Research LaboratoryVDRL, da marca Laboclin ${ }^{\circledR}$.

Para a detecção do marcador do T. cruzy, o anticorpo antitripanossoma é realizado o teste imunoenzimático enzyme immunoassay- EIA, pela marca Ebram ${ }^{\circledR}$.

\section{ANÁLISE ESTATÍSTICA}

Para o tratamento estatístico dos dados foi utilizado o programa Epidata Analysis. ${ }^{11}$

Foram calculadas proporções e médias com seus respectivos intervalos de confiança de $95 \%(p<0,05)$. Foi realizado o teste do qui-quadrado entre as sorologias e as variáveis idade e gênero, distribuídas por ano, com vistas a identificar os fatores associados às soroprevalências dos marcadores das doenças. Para a comparação das médias, foi utilizado o teste T de Student. Aplicou-se o teste de Bartlett para testar a homogeneidade de variância.

O estudo foi realizado de acordo com as diretrizes éticas da pesquisa com seres humanos, recomendadas pelo Conselho Nacional de Pesquisa (CONEP). O projeto foi aprovado pelo Comitê de Ética em Pesquisa da Secretaria de Estado da Saúde de Santa Catarina, com Certificado de Apresentação para Apreciação Ética número 00030363242-08 e folha de rosto número 193746 emitido em agosto de 2008 .

\section{RESULTADOS}

A população deste estudo constituiu-se de 1424 potenciais doadores de órgãos analisados. Destes, 551 (38,7\%) pertencem ao gênero feminino e a maioria, $873(61,3 \%)$ ao gênero masculino. Verificou-se um aumento no número de potenciais doadores em ambos os gêneros entre 2001 e 2007, entretanto manteve-se a maior frequência do gênero masculino (Tabela 1). Também houve um aumento estatisticamente significativo $(\mathrm{p}<0,001)$ da média de idade dos potenciais doadores no período estudado, de 35 anos em 2001 para 47 anos em 2007. Observou-se ainda um aumento da proporção de potenciais doadores na faixa etária com 60 anos e mais em relação às outras faixas etárias, tanto de homens como de mulheres.

$\mathrm{Na}$ Tabela 2, encontram-se os resultados das sorologias para cada marcador no período estudado. O percentual de sorologias positivas para o marcador anti-HBc aumentou de 3,7\% em 2001 para 25,9\% em 2007. Para o marcador HBsAg houve 3,9\% de sorologias positivas no período, variando entre 1,4\% em 2005 e $5,9 \%$ em 2006. Em relação ao anti-HCV, não houve casos positivos em 2001 e 2002 , enquanto $1,3 \%$ dos casos foram positivos entre 2003 e 2007.

Para doença de Chagas, apenas quatro $(0,3 \%)$ sorologias foram positivas entre 1422 doadores testados no período, sendo que nenhuma destas de 2002 a 2006.

Os resultados para sífilis também mostraram-se estáveis ao longo do período, com $0,9 \%$ de soropositividade dos 1423 potenciais doadores de órgãos testados, sendo que nos anos de 2002 e 2003 não houve resultado positivo. Para o HTLV I/II, a soropositividade do período foi baixa, apenas quatro casos $(0,3 \%$ da população de 1420 potenciais doadores de órgãos). Em relação ao HIV I/ II, apenas sete casos soropositivos $(0,5 \%)$ foram encontrados em 1412 testes realizados, sendo que nos anos de 2001, 2002 e 2004 não houve nenhum caso positivo. Com relação à toxoplasmose, como o exame não era rotina no processo de triagem antes de 2006, apenas 888 testes foram realizados para o marcador toxo IgG e destes 38,2\% apresentaram sorologia positiva. Em relação ao marcador toxo IgM, num total de 883 sorologias testadas, apenas $2,3 \%$, apresentaram soropositividade no período estudado. Os resultados dos testes para citomegalovírus foram de: $46,2 \%$ de soropositividade para o marcador CMV IgG, em 1408 potenciais doadores e 2,6\% soropositivos para o marcador CMV IgM em 1351 potenciais doadores de órgãos no período.

$\mathrm{Na}$ Tabela 3, encontram-se os resultados dos marcadores segundo gênero. $\mathrm{O}$ marcador anti-HBc foi mais frequente nos homens. Para o marcador HBsAg a soropositividade também foi maior entre homens, com diferença estatisticamente limítrofe $(p=0,0797)$. Em relação à hepatite $\mathrm{C}$, não houve diferença entre os gêneros. Quanto ao HIV I/II, todos os sete casos $(0,8 \%)$ foram detectados em homens e em relação ao HTLV I/II as sorologias foram semelhantes em homens e mulheres. Para citomegalovírus, ambos os marcadores (CMV $\operatorname{IgG} / \operatorname{IgM}$ ) foram mais prevalentes em mulheres, sendo a diferença estatisticamente significativa apenas para CMV $\operatorname{IgM}$ $(\mathrm{p}=0,0025)$.

Os resultados dos testes sorológicos para sífilis foram positivos em $1,1 \%$ das mulheres e $0,8 \%$ dos homens. Para Chagas, a sorologia positiva foi de $0,2 \%$ nas mulheres e $0,3 \%$ nos homens. Para a toxoplasmose, o marcador IgG teve $35,5 \%$ de sorologias positivas em mulheres e 39,9\% em homens, enquanto para o marcador IgM foram 1,7\% em mulheres e 2,6\% em homens (diferenças não significativas).

Na Tabela 4, estão os resultados para cada marcador, segundo a faixa etária. Para o marcador anti-HBc, o aumento de percentual de sorologias positivas de $3,4 \%$ nos menores de 10 anos para $31,0 \%$ na faixa etária acima de 60 anos foi estatisticamente significativo 
$(\mathrm{p}=0.001)$. Para o marcador HBsAg, houve maior prevalência de soropositivos na faixa etária de 10 a 19 anos, porém sem diferença significativa entre as faixas etárias. Para o anti-HCV, a maior prevalência encontrada foi nos indivíduos de 30 a 39 anos (4,3\%). Para HIV I/II a maior prevalência de resultados positivos foi entre 40 e 49 anos (1,4\%). Para o marcador HTLV I/II, a faixa etária com maior positividade foi 30 a 39 anos $(0,6 \%)$. Em relação ao citomegalovírus ambos os marcadores apresentaram maior soropositividade em crianças e jovens do que em adultos acima de 50 anos e idosos.

Quanto a sífilis a faixa etária de maior soropositividade foi a de 40 a 49 anos $(2,1 \%)$. Para doença de Chagas foram encontrados resultados positivos apenas em duas faixas etárias: 40 a 49 anos e acima de 60. Em relação à toxoplasmose, para o marcador toxo $\mathrm{IgG}$, houve aumento significativo da soropositividade com o aumento da idade $(p=0,0105)$. A faixa etária de maior soropositividade foi a de 60 anos ou mais (44,3\%). Para o marcador toxo IgM, a faixa etária de maior soropositividade foi de 10 a 19 anos.

Observou-se que a frequência de resultados indeterminados e inconclusivos ficou abaixo de $10 \%$ para todas as doenças, exceto para toxoplasmose, que apresentou $37,1 \%$ de resultados inconclusivos para (toxo $\mathrm{IgG}$ ) e $37,2 \%$ de resultados inconclusivos para toxo IgM.

\section{DISCUSSÃO}

A triagem sorológica em bancos de sangue difere da triagem para doação de órgãos sólidos porque não se faz o teste confirmatório e nos transplantes é realizada a triagem sorológica sem a realização da triagem clínica. Os testes são sensíveis, acarretando muitos falsos-positivos e descartes desnecessários na doação de órgãos.

No presente estudo, a maioria dos potenciais doadores de órgãos foi composta de indivíduos do gênero masculino. Essa observação foi relatada num estudo semelhante em potenciais doadores de órgãos sólidos. ${ }^{6}$ Há também outro estudo que traz uma abordagem semelhante, porém em banco de sangue com $83,6 \%$ de homens para $16,4 \%$ de mulheres. ${ }^{12}$ Em outro estudo de transplantes de órgãos houve a predominância do gênero masculino $(60 \%){ }^{13}$ Há semelhança também em outro estudo de soroprevalência de doenças no Pará, em relação ao gênero onde a maioria dos indivíduos que foram reagentes a algum marcador sorológico em todos os anos analisados, eram homens. ${ }^{14}$

O número de potenciais doadores aumentou consideravelmente de 2001 a 2007. Os dados na série histórica temporal também apontam um aumento das médias de idade da população de potenciais doadores. Este aumento também foi verificado em outros estudos. ${ }^{15}$ $\mathrm{O}$ crescimento do número de potenciais doadores de órgãos e consequentemente, o aumento do número de doações efetivadas, pode estar relacionado às campanhas de informação sobre o sistema de transplantes. Segundo dados da Associação Brasileira de Transplantes de Órgãos (ABTO), há aumento progressivo na realização dos principais transplantes de órgãos sólidos no país e o Brasil começa a destacar-se no cenário dos transplantes de órgãos no mundo.

Neste estudo, verificou-se um aumento de potenciais doadores de órgãos nas faixas etárias mais elevadas, especialmente com 60 anos. Sugere-se que, em semelhança às tendências presentes na população geral da maioria dos países, há um aumento de doadores idosos em transplantes. ${ }^{15}$ Essas mudanças podem ter relação com a convergência demográfica e com os avanços da medicina, além de campanhas de conscientização da saúde da população brasileira. ${ }^{16}$

$\mathrm{O}$ gênero mais atingido pela hepatite $\mathrm{B}$ foi o masculino e a maior prevalência de HBsAg foi observada entre 10 e 19 anos. Estudo semelhante revelou que o gênero mais atingido pela hepatite $B$ foi o masculino e a maior incidência de hepatite $B$ foi observada na faixa etária dos 20 aos 49 anos para Santa Catarina e acima dos 30 anos para o Brasil. ${ }^{17}$

Em um estudo realizado em Portugal, observou-se que a maioria dos casos de hepatite B (83\%) eram em homens com idade situada entre os 15 e 45 anos. $^{18} \mathrm{O}$ aumento na soroprevalência desse marcador em países da América Latina, a partir dos 16 anos de idade, sugere a transmissão sexual como maior rota de infecção. A prevalência maior no gênero masculino em relação ao gênero feminino está relacionada possivelmente ao estilo de vida e aos comportamentos que oferecem maior risco, como o uso de drogas injetáveis e relações sexuais hetero e homossexuais sem uso de preservativos, sugerindo a transmissão sexual como maior rota de infecção. ${ }^{19} \mathrm{~A}$ introdução da vacina contra a hepatite $\mathrm{B}$ e a aplicação geral na infância, conduziram vários países a uma redução do número de crianças com infecção crônica. Apesar disto, Amaral et al. observou que a soroprevalência em doadores de órgãos do marcador HBsAg foi de $1,8 \%$ e do marcador anti-HBc foi de $23,8 \%{ }^{6}$

Neste estudo, para o marcador da hepatite $\mathrm{C}$ (anti-HCV), foram encontrados 19 casos de soropositividade (1,3\%). Amaral et al. relataram $2,3 \%$ de positividade entre as notificações de potenciais doadores de órgãos. ${ }^{6}$ Uma pesquisa realizada nos EUA detectou $1,8 \%$ de soropositividade em 716 doadores de órgãos que desenvolveram a doença 3,8 meses depois do transplante. ${ }^{20}$

Neste estudo, os únicos sete casos de soropositivos para o HIV foram do gênero masculino. ${ }^{21}$ Estudos revelam que a infecção está em expansão entre as mulheres, porém ainda a soroprevalência é maior em homens. Segundo D’Orsi, no decorrer dos anos, a incidência no gênero feminino vem aumentando em Santa Catarina, diminuindo a razão de masculinidade. ${ }^{22} \mathrm{O}$ aumento da prevalência da infecção com o avanço da idade neste e em outros estudos pode estar associado com as populações nas quais os mecanismos de transmissão mais importantes envolvem aspectos comportamentais adquiridos ao longo da vida, principalmente a atividade sexual de risco. ${ }^{23,24}$

Apenas quatro $(0,3 \%)$ dos candidatos à doação apresentaram positividade para doença de Chagas neste estudo. Esse resultado é semelhante ao do Amaral et al., onde foi encontrado apenas um caso soropositivo (autóctone) em candidatos à doação de órgãos no estado. ${ }^{6}$ Existem poucos trabalhos publicados sobre a doença de Chagas em Santa Catarina, devido à baixa endemicidade. Porém, é a infecção protozoária intracelular mais frequente na América Latina e tem sido descrita desde o sul dos Estados Unidos até o sul da Patagônia, representando um grande problema de saúde pública. Problemas econômicos e políticos favorecem a migração da doença de Chagas dos países endêmicos para os países desenvolvidos. ${ }^{25} \mathrm{~A}$ transmissão do T. cruzi por meio do transplante renal documentada na literatura e os possíveis efeitos da imunossupressão sobre a infecção crônica excluíram durante muitos anos os indivíduos com sorologia positiva para doença de Chagas como potenciais doadores de órgãos para transplantes. Entretanto, a alta prevalência dessa infecção endêmica na América do Sul e a falta de doadores falecidos estimulou relatos na literatura de transplantes renais utilizando esses indivíduos com sorologia positiva como doadores de órgãos. Os resultados ainda são conflitantes, pois alguns autores apresentam relatos sem evidências de transmissão do parasita e são favoráveis a essa política, enquanto outros mostram o desenvolvimento da doença na sua forma aguda no período póstransplante. ${ }^{26,27} \mathrm{~A}$ redução da prevalência significa, de um lado, a própria redução da incidência da esquizotripanose, geralmente por conta da redução da transmissão vetorial do parasito, devido principalmente à eficácia das ações de controle dos triatomíneos. Essa situação de redução da soropositividade chagásica tem mantido-se em declínio há pelo menos três décadas. ${ }^{27}$

Quanto à sífilis, neste estudo foram encontrados $13(0,9 \%)$ dos potenciais doadores de órgãos com sorologia positiva para essa 
infecção. Amaral et al. observaram 1,6 sorologias positivas em candidatos à doação de órgãos. ${ }^{6} \mathrm{O}$ advento da terapia com antibióticos mudou o cenário da infecção sifilítica, possibilitando a cura, a interrupção da sua evolução e a diminuição considerável da sua mortalidade. ${ }^{28}$ Porém, apesar da prevalência da sorologia positiva para VDRL ter diminuído nos últimos anos, alguns trabalhos mostram um aumento na prevalência de sífilis em vários países. $^{?}$

Estudos realizados nos Estados Unidos apontam que a sífilis continua a crescer entre os homens que fazem sexo com homens, enquanto mantém-se estável entre as mulheres. ${ }^{29}$ Porém, doadores de sangue possuem características diferentes da população de doadores de órgãos. A transmissibilidade da sífilis em doadores de sangue é confirmada, mas para as doações de órgãos sólidos, não há evidências quanto à sua transmissão aos enxertos oriundos de cadáveres. $\mathrm{O}$ conhecimento do perfil sorológico em pacientes transplantados para sífilis é essencial para adequar aos soropositivos que consiste principalmente do uso de penicilina. ${ }^{30}$

Quanto à infecção por HTLV I/II, o presente estudo verificou a prevalência total da população pesquisada em apenas quatro casos $(0,3 \%)$. Entre os potenciais doadores de órgãos sólidos pesquisados por Amaral et al., não houve casos de infecção pelo vírus em doadores de órgãos. ${ }^{6}$

Estudos sugerem a relação entre maior idade e maior risco de infecção, particularmente para as mulheres, cujo aumento da susceptibilidade pode ser atribuído ao aumento de exposições sexuais com parceiros infectados ou mudanças biológicas em função de alterações hormonais. ${ }^{31}$ Um estudo realizado no Japão confirma a suscetibilidade da infecção pelo HTLVI, tendo sido especialmente maior em mulheres mais velhas quando o risco da transmissão do HTLV I foi maior em mulheres que se encontravam em período pós-menopausa. ${ }^{31}$ No Brasil, as taxas entre os doadores de sangue apontam a prevalência mais alta em Salvador. ${ }^{32}$ Para a infecção causada pelo vírus HTLV II, estudos relatam a sua endemicidade em populações indígenas das Américas. ${ }^{32}$

Em um estudo conduzido na China, foram sorologicamente testados $68 \%$ da população acima de 30 anos e num total de 3010 voluntários, as mulheres apresentaram soroprevalência de $0,87 \% \mathrm{e}$ os homens de $0,56 \% .{ }^{33}$ Num estudo conduzido em Guiné Bissau, prevalências foram mais elevadas em homens $(4,6 \%)$ e mulheres $(12,4 \%)$ com mais de 50 anos. ${ }^{34}$ Nos Estados Unidos, estudos em áreas endêmicas têm mostrado que a soroprevalência está associada com idade e gênero. ${ }^{34}$

Quanto à sorologia de CMV IgG, este estudo apontou uma alta prevalência na população, pois quase a metade, ou seja $650(46,2 \%)$ dos potenciais doadores apresentaram sorologia positiva para essa infecção. A faixa etária com maior frequência de sorologias positivas para CMV IgG foi em menores de 10 anos, sugerindo maior prevalência dessa doença em jovens.

Quanto ao marcador CMV IgM, que indica infecção recente, neste estudo observou-se 2,6\% de soroprevalência. Amaral ET al. encontraram prevalência em potenciais doadores de órgãos sólidos, para o período de 2006 a 2007 de apenas $1,1 \%{ }^{6}{ }^{6}$

Nos Estados Unidos, o CMV está presente em todas as regiões em adultos acima de 40 anos de idade..$^{35} \mathrm{O}$ citomegalovírus transmitido através de órgãos sólidos causa infecção e doença nos receptores, especialmente nos receptores CMV negativos. ${ }^{36}$ Estudos relatam que pacientes submetidos ao transplante de medula óssea têm alta mortalidade quando desenvolvem citomegalovirose. ${ }^{36}$ Vários estudos epidemiológicos têm sugerido uma relação entre o risco de infecção pelo citomegalovírus CMV em pacientes queimados e a utilização de aloenxertos com sorologia positiva para CMV. ${ }^{37}$ A infecção por CMV contribui para o aumento da morbidade e mortalidade destes pacientes.
Os processos da doença relacionada ao CMV manifestam-se de maneira diferente, dependendo de qual é o órgão transplantado. Em receptores de medula óssea, a infecção por CMV ocorre como uma pneumonia intersticial, com alta taxa de mortalidade. Em receptores de fígado, a hepatite pode ser problemática e difícil de ser diferenciada de uma rejeição do órgão. ${ }^{38}$

A apresentação da "síndrome do CMV", que consiste em febre, leucopenia, linfócitos atípicos, hepatomegalia, mialgia e artralgia, é a manifestação mais comum da infecção primária por $\mathrm{CMV}$ em pacientes que receberam transplante de rim. ${ }^{39}$

A infecção por CMV é, certamente, a mais comum infecção em órgãos transplantados e pode ser responsabilizada por $30 \%$ dos episódios de febre, $35 \%$ de toda leucopenia e $20 \%$ de toda chance de falha do transplante do órgão.?

A importância do conhecimento do perfil sorológico dá-se em razão da profilaxia adequada contra $\mathrm{CMV}$, para diminuir a morbimortalidade associada à infecção por esse vírus.

Em relação à toxoplasmose, somente no final do ano de 2005, a sorologia para essa doença passou a ser rotina no processo de triagem ${ }^{6}$ e, dessa forma, apenas os dados referentes a 2006 e 2007 representam o total de doadores testados neste trabalho. $\mathrm{Na}$ sorologia para toxo IgG verificou-se aumento desse marcador em pessoas mais velhas.

No trabalho de Amaral et al., o marcador toxo IgM foi de 1,64\%, abaixo do valor encontrado neste estudo, que foi de $2,3 \%{ }^{6} \mathrm{~A}$ provável explicação para a diferença dos dados de prevalência encontrados por Amaral et al. é o período de estudo, que foi de apenas dois anos, enquanto que neste estudo o período foi de seis anos. A presença do marcador para essa infecção é preocupante nos transplantes cardíacos, pois o protozoário pode se alojar nesse músculo. ${ }^{40}$ A importância clínica dessa infecção é evidenciada em indivíduos imunocomprometidos, como pacientes transplantados que estão sob terapia imunossupressora, onde há a possibilidade de reativação da infecção crônica, a qual pode evoluir para quadro clínico grave, podendo culminar com o óbito. ${ }^{42}$ Esse fato releva a importância do conhecimento do perfil sorológico para toxoplasmose em processos de transplantes de órgãos. ${ }^{41}$

O transplante tornou-se o procedimento de escolha para $o$ tratamento de doenças terminais. $\mathrm{O}$ sucesso do tratamento resultou no aumento da demanda de órgãos, sem o aumento correspondente de sua oferta.

Para enfrentar esse problema, numerosas estratégias têm sido propostas, como o uso de doadores anteriormente considerados inadequados, os doadores limítrofes, que possuem sorologia positiva para algum dos marcadores de doenças. Esse recurso tem se mostrado viável para aumentar a oferta de órgãos para transplantes, aliado à profilaxia adequada a cada perfil sorológico.

\section{CONCLUSÃO}

Houve maior prevalência do gênero masculino entre os potenciais doadores de órgãos e aumento na faixa etária acima de 60 anos. $\mathrm{O}$ marcador HBsAg foi maior em homens durante o período estudado. As soroprevalências de HCV, HIV I/II, HTLV I/II e doença de Chagas foram baixas, sem alterações ao longo do período estudado.

As infecções por citomegalovírus e toxoplasmose mostraram-se elevadas, sendo a infecção aguda pouco comum. Citomegalovírus foi mais frequente nas mulheres e nos mais jovens. O conhecimento da distribuição das doenças nos potenciais doadores é importante para a compreensão do risco de transmissão na doação de órgãos sólidos. 
Tabela 1: Distribuição de candidatos à doação de órgãos segundo gênero, faixa etária e ano, Santa Catarina (2001-2007)

\begin{tabular}{|c|c|c|c|c|c|c|c|c|c|c|c|c|c|c|c|c|}
\hline \multirow[b]{2}{*}{ MASCULINO } & \multicolumn{2}{|c|}{2001} & \multicolumn{2}{|c|}{2002} & \multicolumn{2}{|c|}{2003} & \multicolumn{2}{|c|}{2004} & \multicolumn{2}{|c|}{2005} & \multicolumn{2}{|c|}{2006} & \multicolumn{2}{|c|}{2007} & \multicolumn{2}{|c|}{ Total } \\
\hline & $\mathrm{N}$ & $\%$ & $\mathrm{~N}$ & $\%$ & $\mathrm{~N}$ & $\%$ & $\mathrm{~N}$ & $\%$ & $\mathrm{~N}$ & $\%$ & $\mathrm{~N}$ & $\%$ & $\mathrm{~N}$ & $\%$ & $\mathrm{~N}$ & $\%$ \\
\hline 0 a 9 & 2 & 6.3 & 2 & 4.9 & 4 & 6.0 & 0 & 0.0 & 2 & 1.6 & 6 & 2.3 & 3 & 1.1 & 19 & 2.2 \\
\hline 10 a 19 & 6 & 18.8 & 7 & 17.1 & 10 & 14.9 & 9 & 13.0 & 14 & 10.9 & 19 & 7.4 & 24 & 8.7 & 89 & 10.2 \\
\hline 20 a 29 & 6 & 18.8 & 7 & 17.1 & 15 & 22.4 & 14 & 20.3 & 28 & 21.7 & 45 & 17.6 & 54 & 19.6 & 169 & 19.4 \\
\hline 30 a 39 & 4 & 12.5 & 11 & 26.8 & 10 & 14.9 & 4 & 5.8 & 15 & 11.6 & 17 & 6.6 & 36 & 13.0 & 97 & 11.1 \\
\hline 40 a 49 & 7 & 21.9 & 5 & 12.2 & 11 & 16.4 & 19 & 27.5 & 22 & 17.1 & 54 & 21.1 & 34 & 12.3 & 152 & 17.5 \\
\hline 50 a 59 & 4 & 12.5 & 7 & 17.1 & 14 & 20.9 & 11 & 15.9 & 22 & 17.1 & 48 & 18.8 & 61 & 22.1 & 167 & 19.2 \\
\hline $60+$ & 3 & 9.4 & 2 & 4.9 & 3 & 4.5 & 12 & 17.4 & 26 & 20.2 & 67 & 26.2 & 64 & 23.2 & 177 & 20.3 \\
\hline Total Masculino & 32 & 100.0 & 41 & 100.0 & 67 & 100.0 & 69 & 100.0 & 129 & 100.0 & 256 & 100.0 & 276 & 100.0 & 870 & 100.0 \\
\hline Média (DP) & 35,75 & $19,62)$ & 34.37 & 14.75) & 34.76( & 16.93) & 41.42( & 15.97) & 41.83 & 19.10) & 45.94 & 20.20) & 44.57 & (18.58) & 42.72( & 19.05) \\
\hline $\begin{array}{l}\text { Sub-total } \\
\text { Masculino }\end{array}$ & & & 67 & & 65 & & 57. & & 58 & & 62 & & 60 & .7 & 61 & .2 \\
\hline
\end{tabular}

\begin{tabular}{|c|c|c|c|c|c|c|c|c|c|c|c|c|c|c|c|c|}
\hline FEMININO & $\mathrm{n}$ & $\%$ & $\mathrm{n}$ & $\%$ & $\mathrm{~N}$ & $\%$ & $\mathrm{~N}$ & $\%$ & $\mathrm{n}$ & $\%$ & $\mathrm{n}$ & $\%$ & $\mathrm{~N}$ & $\%$ & $\mathrm{n}$ & $\%$ \\
\hline 0 a 9 & 1 & 4.5 & 2 & 10.0 & 2 & 5.7 & 2 & 3.9 & 5 & 5.4 & 4 & 2.6 & 1 & 0.6 & 17 & 3.1 \\
\hline 10 a 19 & 2 & 9.1 & 2 & 10.0 & 4 & 11.4 & 8 & 15.7 & 2 & 2.2 & 10 & 6.6 & 8 & 4.5 & 36 & 6.5 \\
\hline 20 a 29 & 3 & 13.6 & 1 & 5.0 & 4 & 11.4 & 3 & 5.9 & 9 & 9.8 & 7 & 4.6 & 20 & 11.2 & 47 & 8.5 \\
\hline 30 a 39 & 6 & 27.3 & 6 & 30.0 & 7 & 20.0 & 8 & 15.7 & 6 & 6.5 & 11 & 7.2 & 21 & 11.7 & 65 & 11.8 \\
\hline 40 a 49 & 7 & 31.8 & 6 & 30.0 & 7 & 20.0 & 13 & 25.5 & 31 & 33.7 & 38 & 25.0 & 31 & 17.3 & 133 & 24.1 \\
\hline 50 a 59 & 3 & 13.6 & 1 & 5.0 & 5 & 14.3 & 10 & 19.6 & 19 & 20.7 & 26 & 17.1 & 40 & 22.3 & 104 & 18.9 \\
\hline $60+$ & 0 & 0.0 & 2 & 10.0 & 6 & 17.1 & 7 & 13.7 & 20 & 21.7 & 56 & 36.8 & 58 & 32.4 & 149 & 27.0 \\
\hline Total Feminino & 22 & 100.0 & 20 & 100.0 & 35 & 100.0 & 51 & 100.0 & 92 & 100.0 & 152 & 100.0 & 179 & 100.0 & 551 & 100.0 \\
\hline Média (DP) & 36.14( & (13.20) & 36.9 & 7.74) & 39.63 & 18.56) & 41.22( & 18.34) & 46.61 & (17.75) & 50.77 & $(18.64)$ & 50.89 & (19.74) & 47.44 & (19.15) \\
\hline $\begin{array}{l}\text { Sub-total } \\
\text { Feminino } \\
\end{array}$ & \multicolumn{2}{|c|}{40.7} & \multicolumn{2}{|c|}{32.8} & \multicolumn{2}{|c|}{34.3} & \multicolumn{2}{|c|}{42.5} & \multicolumn{2}{|c|}{41.6} & \multicolumn{2}{|c|}{37.3} & \multicolumn{2}{|c|}{39.3} & \multicolumn{2}{|c|}{38.8} \\
\hline Total geral ${ }^{\star}$ & 54 & 100,0 & 61 & 100,0 & 102 & 100,0 & 120 & 100,0 & 221 & 100,0 & 408 & 100,0 & 455 & 100,0 & 1421 & 100,0 \\
\hline
\end{tabular}

\footnotetext{
* Excluídos os casos com idade ignorada $(\mathrm{n}=3)$.

** desvio padrão
} 
Tabela 2: Distribuição de candidatos à doação de órgãos segundo gênero, faixa etária e ano, Santa Catarina (2001-2007)

\begin{tabular}{|c|c|c|c|c|c|c|c|c|c|c|c|c|c|c|c|c|}
\hline & \multicolumn{2}{|c|}{2001} & \multicolumn{2}{|c|}{2002} & \multicolumn{2}{|c|}{2003} & \multicolumn{2}{|c|}{2004} & \multicolumn{2}{|c|}{2005} & \multicolumn{2}{|c|}{2006} & \multicolumn{2}{|c|}{2007} & \multicolumn{2}{|c|}{ Total } \\
\hline & $\mathbf{N}$ & $\%$ & $\mathbf{N}$ & $\%$ & $\mathbf{N}$ & $\%$ & $\mathbf{N}$ & $\%$ & $\mathbf{N}$ & $\%$ & $\mathbf{N}$ & $\%$ & $\mathbf{N}$ & $\%$ & $\mathbf{N}$ & $\%$ \\
\hline \multicolumn{17}{|l|}{ Anti-HBc } \\
\hline Negativo & 52 & 96,3 & - & - & 56 & 87,5 & 102 & 85,7 & 178 & 81,7 & 301 & 74,3 & 340 & 75,1 & 1029 & 78,3 \\
\hline Positivo & 2 & 3,7 & 1 & 100,0 & 8 & 12,5 & 17 & 14,3 & 40 & 18,3 & 104 & 25,7 & 113 & 24,9 & 285 & 21,7 \\
\hline Total & 54 & 100,0 & 1 & 100,0 & 64 & 100,0 & 119 & 100,0 & 218 & 100,0 & 405 & 100,0 & 453 & 100,0 & 1314 & 100,0 \\
\hline \multicolumn{17}{|l|}{ HBsAg } \\
\hline Negativo & 53 & 98,1 & 61 & 98,4 & 102 & 100,0 & 113 & 95,0 & 219 & 98,6 & 381 & 94,1 & 435 & 95,6 & 1364 & 96,1 \\
\hline Positivo & 1 & 1,9 & 1 & 1,6 & - & - & 6 & 5,0 & 3 & 1,4 & 24 & 5,9 & 20 & 4,4 & 55 & 3,9 \\
\hline Total & 54 & 100,0 & 62 & 100,0 & 102 & 100,0 & 119 & 100,0 & 222 & 100,0 & 405 & 100,0 & 455 & 100,0 & 1419 & 100,0 \\
\hline \multicolumn{17}{|c|}{ Anti-HCV } \\
\hline Negativo & 54 & 100,0 & 62 & 100,0 & 100 & 98,0 & 119 & 99,2 & 217 & 98,2 & 401 & 99,0 & 447 & 98,2 & 1400 & 98,7 \\
\hline Positivo & - & - & - & - & 2 & 2,0 & 1 & 0,8 & 4 & 1,8 & 4 & 1,0 & 8 & 1,8 & 19 & 1,3 \\
\hline Total & 54 & 100,0 & 62 & 100,0 & 102 & 100,0 & 120 & 100,0 & 221 & 100,0 & 405 & 100,0 & 455 & 100,0 & 1419 & 100,0 \\
\hline \multicolumn{17}{|l|}{ Chagas } \\
\hline Negativo & 53 & 98,1 & 62 & 100,0 & 102 & 100,0 & 120 & 100,0 & 222 & 100,0 & 407 & 100,0 & 452 & 99,3 & 1418 & 99,7 \\
\hline Positivo & 1 & 1,9 & - & - & - & - & - & - & - & - & - & - & 3 & 0,7 & 4 & 0,3 \\
\hline Total & 54 & 100,0 & 62 & 100,0 & 102 & 100,0 & 120 & 100,0 & 222 & 100,0 & 407 & 100,0 & 455 & 100,0 & 1422 & 100,0 \\
\hline \multicolumn{17}{|l|}{ Sífilis } \\
\hline Negativo & 53 & 98,1 & 62 & 100,0 & 102 & 100,0 & 119 & 99,2 & 221 & 99,5 & 403 & 98,8 & 450 & 98,9 & 1410 & 99,1 \\
\hline Positivo & 1 & 1,9 & - & - & - & - & 1 & 0,8 & 1 & 0,5 & 5 & 1,2 & 5 & 1,1 & 13 & 0,9 \\
\hline Total & 54 & 100,0 & 62 & 100,0 & 102 & 100,0 & 120 & 100,0 & 222 & 100,0 & 408 & 100,0 & 455 & 100,0 & 1423 & 100,0 \\
\hline \multicolumn{17}{|c|}{ HTLV I/II } \\
\hline Negativo & 53 & 98,1 & 62 & 100,0 & 102 & 100,0 & 120 & 100,0 & 220 & 99,5 & 406 & 99,8 & 453 & 99,8 & 1416 & 99,7 \\
\hline Positivo & 1 & 1,9 & - & - & - & - & - & - & 1 & 0,5 & 1 & 0,2 & 1 & 0,2 & 4 & 0,3 \\
\hline Total & 54 & 100,0 & 62 & 100,0 & 102 & 100,0 & 120 & 100,0 & 221 & 100,0 & 407 & 100,0 & 454 & 100,0 & 1420 & 100,0 \\
\hline \multicolumn{17}{|l|}{ HIV I/II } \\
\hline Negativo & 54 & 100,0 & 62 & 100,0 & 101 & 99,0 & 118 & 100,0 & 218 & 99,1 & 402 & 99,5 & 450 & 99,6 & 1405 & 99,5 \\
\hline Positivo & - & - & - & - & 1 & 1 & - & - & 2 & 0,9 & 2 & 0,5 & 2 & 0,4 & 7 & 0,5 \\
\hline Total & 54 & 100,0 & 62 & 100,0 & 102 & 100,0 & 118 & 100,0 & 220 & 100,0 & 404 & 100,0 & 452 & 100,0 & 1412 & 100,0 \\
\hline \multicolumn{17}{|l|}{ Toxo IgG } \\
\hline Negativo & 47 & 87,0 & - & - & 1 & 100,0 & 31 & 100,0 & - & - & 293 & 83,5 & 177 & 39,3 & 549 & 61,8 \\
\hline Positivo & 7 & 13,0 & - & - & - & - & - & - & 1 & 100,0 & 58 & 16,5 & 273 & 60,7 & 339 & 38,2 \\
\hline Total & 54 & 100,0 & - & - & 1 & 100,0 & 31 & 100,0 & 1 & 100,0 & 351 & 100,0 & 450 & 100,0 & 888 & 100,0 \\
\hline \multicolumn{17}{|l|}{ Toxo IgM } \\
\hline Negativo & 52 & 96,3 & - & - & 1 & 100,0 & 31 & 100,0 & - & - & 341 & 99,4 & 438 & 96,5 & 863 & 97,7 \\
\hline Positivo & 2 & 3,7 & - & - & - & - & - & - & - & - & 2 & 0,6 & 16 & 3,5 & 20 & 2,3 \\
\hline Total & 54 & 100,0 & - & - & 1 & 100,0 & 31 & 100,0 & - & - & 343 & 100,0 & 454 & 100,0 & 883 & 100,0 \\
\hline \multicolumn{17}{|l|}{ CMV IgG } \\
\hline Negativo & 50 & 92,6 & 22 & 37,3 & 61 & 62,2 & 65 & 55,1 & 112 & 51,1 & 201 & 49,5 & 247 & 54,4 & 758 & 53,8 \\
\hline Positivo & 4 & 7,4 & 37 & 62,7 & 37 & 37,8 & 53 & 44,9 & 107 & 48,9 & 205 & 50,5 & 207 & 45,6 & 650 & 46,2 \\
\hline Total & 54 & 100,0 & 59 & 100,0 & 98 & 100,0 & 118 & 100,0 & 219 & 100,0 & 406 & 100,0 & 454 & 100,0 & 1408 & 100,0 \\
\hline CMV IgM & & & & & & & & & & & & & & & & \\
\hline Negativo & 52 & 98,1 & 57 & 98,3 & 81 & 98,8 & 106 & 92,2 & 212 & 97,2 & 362 & 96,5 & 446 & 99,1 & 1316 & 97,4 \\
\hline Positivo & 1 & 1,9 & 1 & 1,7 & 1 & 1,2 & 9 & 7,8 & 6 & 2,8 & 13 & 3,5 & 4 & 0,9 & 35 & 2,6 \\
\hline Total & 53 & 100,0 & 58 & 100,0 & 82 & 100,0 & 115 & 100,0 & 218 & 100,0 & 375 & 100,0 & 450 & 100,0 & 1351 & 100,0 \\
\hline
\end{tabular}


Tabela 3: Marcadores sorológicos em doadores de órgãos segundo gênero, Santa Catarina (2001 a 2007)

\begin{tabular}{|c|c|c|c|c|c|c|c|c|c|c|c|c|c|c|c|}
\hline & \multicolumn{2}{|c|}{ Feminino } & \multicolumn{2}{|c|}{ Masculino } & \multicolumn{2}{|c|}{ Total } & \multirow[t]{2}{*}{$\mathbf{P}^{\star}$} & & \multicolumn{2}{|c|}{ Feminino } & \multicolumn{2}{|c|}{ Masculino } & \multicolumn{2}{|c|}{ Total } & \multirow[t]{2}{*}{$\mathbf{P}^{\star}$} \\
\hline & $\mathrm{N}$ & $\%$ & $\mathrm{~N}$ & $\%$ & $\mathrm{~N}$ & $\%$ & & & $\mathrm{~N}$ & $\%$ & $\mathrm{~N}$ & $\%$ & $\mathrm{~N}$ & $\%$ & \\
\hline Anti-HBc & & & & & & & & CMV & & & & & & & \\
\hline Negativo & 413 & 80,4 & 616 & 77,0 & 1029 & 78,3 & & $\operatorname{IgM}$ & & & & & & & \\
\hline Positivo & 101 & 19.6 & 184 & 23,0 & 285 & 21.7 & 0,1504 & Negativo & 496 & 95,8 & 820 & 98,4 & 1316 & 97,4 & \\
\hline Total & 514 & 100,0 & 800 & 100,0 & 1314 & 100,0 & & Positivo & 22 & 4,2 & 13 & 1.6 & 35 & 2.6 & 0,0025 \\
\hline HBsAg & & & & & & & & Total & 518 & 100,0 & 833 & 100,0 & 1351 & 100,0 & \\
\hline Negativo & 532 & 97,3 & 832 & 95.4 & 1364 & 96,1 & & Sífilis & & & & & & & \\
\hline Positivo & 15 & 2.7 & 40 & 4.6 & 55 & 3.9 & 0,0797 & Negativo & 545 & 98.9 & 865 & 99.2 & 1410 & 99.1 & \\
\hline Total & 547 & 100,0 & 872 & 100,0 & 1419 & 100,0 & & Positivo & 6 & 1.1 & 7 & 0.8 & 13 & 0.9 & 0,5805 \\
\hline Anti-HCV & & & & & & & & Total & 551 & 100,0 & 872 & 100,0 & 1423 & 100,0 & \\
\hline Negativo & 544 & 98.9 & 856 & 98.5 & 1400 & 98.7 & & Chagas & & & & & & & \\
\hline Positivo & 6 & 1.1 & 13 & 1.5 & 19 & 1.3 & 0,5178 & Negativo & 549 & 99,9 & 869 & 99.7 & 1418 & 99.7 & \\
\hline Total & 550 & 100,0 & 869 & 100,0 & 1419 & 100,0 & & Positivo & 1 & 0.2 & 3 & 0.3 & 4 & 0.3 & 0,5738 \\
\hline HIV I/II & & & & & & & & Total & 550 & 100,0 & 872 & 100,0 & 1422 & 100,0 & \\
\hline Negativo & 550 & 100,0 & 855 & 99,2 & 1405 & 99,5 & & Toxo IgG & & & & & & & \\
\hline Positivo & - & - & 7 & 0.8 & 7 & 0.5 & - & Negativo & 223 & 64,5 & 326 & 60,1 & 549 & 61.8 & \\
\hline Total & 550 & 100,0 & 862 & 100,0 & 1412 & 100,0 & & Positivo & 123 & 35.5 & 216 & 39.9 & 339 & 38,2 & 0,1980 \\
\hline HTLV I/II & & & & & & & & Total & 346 & 100,0 & 542 & 100,0 & 888 & 100,0 & \\
\hline Negativo & 547 & 99.6 & 869 & 99.8 & 1416 & 99,7 & & Toxo IgM & & & & & & & \\
\hline Positivo & 2 & 0.4 & 2 & 0.2 & 4 & 0.3 & 0,6410 & Negativo & 342 & 98,3 & 521 & 97,4 & 863 & 97,7 & \\
\hline Total & 549 & 100,0 & 871 & 100,0 & 1420 & 100,0 & & Positivo & 6 & 1.7 & 14 & 2.6 & 20 & 2.3 & 0,3836 \\
\hline CMV IgG & & & & & & & & Total & 348 & 100,0 & 535 & 100,0 & 883 & 100,0 & \\
\hline Negativo & 278 & 51,1 & 480 & 55,6 & 758 & 53.8 & & & & & & & & & \\
\hline Positivo & 266 & 48,9 & 384 & 44,4 & 650 & 46,2 & 0,1027 & & & & & & & & \\
\hline Total & 544 & 100,0 & 864 & 100,0 & 1408 & 100,0 & & ${ }^{*} \mathrm{p}=\mathrm{val}$ & este & -quac & & & & & \\
\hline
\end{tabular}

Tabela 4: Percentual de resultados soropositivos ** em doadores de órgãos segundo faixa etária em Santa Catarina (2001-2007)

\begin{tabular}{|c|c|c|c|c|c|c|c|c|c|c|c|c|c|c|c|c|c|c|c|c|c|c|}
\hline \multirow[t]{2}{*}{$\begin{array}{l}\text { Faixa } \\
\text { etária }\end{array}$} & \multicolumn{2}{|c|}{ CMV IgG } & \multicolumn{2}{|c|}{ CMV IgM } & \multicolumn{2}{|c|}{ Toxo IgG } & \multicolumn{2}{|c|}{ Toxo IgM } & \multicolumn{2}{|c|}{ anti-HBc } & \multicolumn{2}{|c|}{ HBsAg } & \multicolumn{2}{|c|}{ HIV I/II } & \multicolumn{2}{|c|}{ HTLV I/II } & \multicolumn{2}{|c|}{ SÍFILIS } & \multicolumn{2}{|c|}{ CHAGAS } & \multicolumn{2}{|c|}{$\begin{array}{l}\text { ANTI } \\
\text {-HCV }\end{array}$} \\
\hline & $\mathrm{N}$ & $\%$ & $\mathrm{~N}$ & $\%$ & $\mathrm{~N}$ & $\%$ & $\mathrm{~N}$ & $\%$ & $\mathrm{~N}$ & $\%$ & $\mathrm{~N}$ & $\%$ & $\mathrm{~N}$ & $\%$ & $\mathrm{~N}$ & $\%$ & $\mathrm{~N}$ & $\%$ & $\mathrm{~N}$ & $\%$ & $\mathrm{~N}$ & $\%$ \\
\hline$<10$ & 34 & 61,8 & 34 & 5,9 & 14 & 21,4 & - & & 29 & 3,4 & 36 & 2,8 & - & & - & & - & & - & & - & \\
\hline 10 a 19 & 122 & 58,2 & 114 & 1,8 & 67 & 26,9 & 66 & 4,5 & 110 & 5,5 & 125 & 6,4 & - & & - & & - & & - & & - & \\
\hline 20 a 29 & 213 & 54,0 & 206 & 2,9 & 134 & 41,8 & 133 & 3,8 & 203 & 11,8 & 216 & 4,2 & 214 & 0,5 & - & & 216 & 0,5 & - & & - & \\
\hline 30 a 39 & 160 & 65,6 & 155 & 1,9 & 92 & 38,0 & 92 & 1,1 & 133 & 19,5 & 162 & 4,9 & - & & 162 & 0,6 & - & & - & & 162 & 4,3 \\
\hline 40 a 49 & 282 & 51,8 & 273 & 5,1 & 166 & 28,9 & 164 & 2,4 & 267 & 21,7 & 283 & 3,2 & 285 & 1,4 & 285 & 0,4 & 285 & 2,1 & 285 & 0,7 & 283 & 1,8 \\
\hline 50 a 59 & 271 & 42,8 & 255 & 1,2 & 180 & 41,7 & 179 & 2,2 & 255 & 28,2 & 269 & 4,5 & 268 & 0,4 & 269 & 0,4 & 271 & 1,1 & - & & 271 & 0,7 \\
\hline $60+$ & 325 & 23,1 & 313 & 1,6 & 235 & 44,3 & 235 & 1,3 & 316 & 31,0 & 326 & 2,5 & 322 & 0,3 & 326 & 0,3 & 326 & 0,9 & 325 & 0,6 & 324 & 1,5 \\
\hline Total & 1407 & 46,1 & 1350 & 2,6 & 888 & 38,2 & 883 & 2,3 & 1313 & 21,7 & 1417 & 3,9 & 1410 & 0,5 & 1418 & 0,3 & 1421 & 0,9 & 1424 & 0,3 & 1417 & 1,3 \\
\hline $\begin{array}{l}\text { Valor } \\
p^{*}\end{array}$ & $<0$, & & 0.05 & & 0.0 & & 0.5 & & $<0,0$ & & 0.5 & & 0.35 & & 0.93 & & 0,2 & & 0,4 & & & \\
\hline
\end{tabular}

${ }^{\star}$ Valor $\mathrm{P}^{\star}$ calculado a partir do qui-quadrado

**Foram excluídos indivíduos indeterminados e inconclusivos 


\section{ABSTRACT:}

Purpose: This study assessed the profile of serological markers of blood-borne diseases among potential donor of solid organs. Methods: We conducted an epidemiological survey based on secondary data. The population studied comprised 1424 potential donors of solid organs in the Santa Catarina State from January 2001 to December 2007. Serological analysis was conducted by the Center for Hematology and Hemotherapy in the Santa Catarina State (HEMOSC). Demographic data were collected by the Center of Organs donation in the state (CNCDO/SC). The variables analyzed include serological test results in potential donors of solid organs, as well as their sex, age and year of examination. Results: Among 1424 subjects examined, 551 (38.7\%) were females and 873 (61.3\%) males. Seroprevalence of disease markers was as follows: $21.7 \%$ with anti-HBc, 3,9\% with $\mathrm{HBsAg}, 1,3 \%$ with anti-HCV, $0,3 \%$ with Chagas disease, $0,9 \%$ with VDRL marker for syphilis, $0,3 \%$ with HTLV 1 or $2,0,5 \%$ with HIV 1 or $2,38.2 \%$ with toxoplasmosis $\operatorname{IgG}, 2,3 \%$ with toxoplasmosis $\operatorname{IgM}, 46,2 \%$ with CMV IgG and 2,6\% with CMV IgM. The seroprevalence of CMV IgM was higher in women $(p<0.01)$. The other markers showed no statistically significant difference between genders. Conclusions: There was a higher percentage of males among potential donors for donation of organs. During the period analyzed, an increase in the percentage of potential donor above 60 years old and HBsAg-positive men was observed. The seroprevalence of HCV, HIV, HTLV and Chagas disease was low with no change along the period studied. Infection by cytomegalovirus and toxoplasmosis were high, and acute infection uncommon. Cytomegalovirus was more frequent in women and younger individuals.

Keywords: Hepatitis B, Hepatitis C, HIV, Human T-lymphotropic vírus-1, Chagas Disease, Cytomegalovirus, Syphilis, Toxoplasmosis, Seroepidemiologic Studies, Organ Transplantation.

\section{REFERÊNCIAS:}

1. Chavalitdhamrong D, Gill J, Takemoto S, Madhira BR, Cho YW, Shah $\mathrm{T}$, et al. Patient and graft outcomes from deceased kidney donors age 70 years and older: an analysis of the Organ Procurement Transplant Network/ United Network of Organ Sharing database. Transplantation. 2008 Jun 15;85(11):1573-9.

2. Herruzo JAS. Tratado de trasplantes de órganos. Rev esp enferm dig [serial on the Internet]. 2006; 98(7): Available from: http://scielo.isciii.es/scielo. php?script=sci_arttext\&pid=S1130-01082006000700016\&lng=en\&nrm=iso.

3. Pereira WA. VI Reunião de Diretrizes Básicas para Captação e Retirada de Múltiplos Órgãos e Tecidos da Associação Brasileira de Transplante de Órgãos 2007. São Paulo: Associação Brasileira de Transplantes de Órgãos 2007.

4. Garcia VD, Pereira WA, Paula FJ. RBT - Registro Brasileiro de Transplantes. São Paulo: Associação Brasileira de Transplante de Órgãos; 2006.

5. Históricos dos Transplantes. [home-page] Florianópolis: CNCDO/SC.SC Transplantes; 2007; Available from: http://sctransplantes.saude.sc.gov.br/index. php?option=com_content $\&$ task $=$ category\&sectionid $=10 \& i d=34 \&$ Itemid $=177$.

6. Amaral RP, Saidneuy AE, Ribeiro WL, Andrade J. Serological profile of potential solid organ donors in Santa Catarina, Brazil. Transplant Proc. 2008;Apr;40(3):665-7.

7. Delmonico F. Cadaver donor screening for infectious agents in solid organ transplantation. Clin Infect Dis. 2000;Sep;31(3):781-6.

8. Ojo A, Hansom J, Meier K. H et al. Survival in recipients of marginal cadaveric donor kidneys compared with other recipients and wait-listed transplant candidadtes. J Am Soc Nephrol. 2001;12:589-97.

9. Garrity EJ, Boettcher H, Gabbay E. Donor infection: an opinion on lung donor utilization. J Heart Lung Transplant. 2005;24:791-7

10. Rosengard BR, Feng S, Alfrey EJ, Zaroff JG, Emond JC, Henry ML, et al. Report of the Crystal City meeting to maximize the use of organs recovered from the cadaver donor. Am J Transplant. 2002;2(8):701-11.

11. EpiData Software. Versão 3.1. 2008. http://www.epidata.dk.

12. Valente VB, Tadeu CD, Passos ADC. Marcadores sorológicos das hepatites $\mathrm{B}$ e $\mathrm{C}$ em doadores de sangue do Hemocentro de Ribeirão Preto, SP. Rev Soc Bras Med Trop. 2005;38(6):488-92.

13. Boaz MR, Bordignon S, Nesralla IA. A importância de medidas preventivas na profilaxia de infecções em pacientes submetidos a transplante cardíaco nos primeiros 30 dias de pós-operatório. Rev Bras Cir Cardiovasc. 2006;21(2):188-93.
14. Aquino JA, Pegado KA, Barros LP, Machado LFA. Soroprevalência de infecções por vírus da hepatite $\mathrm{B}$ e vírus da hepatite $\mathrm{C}$ em indivíduos do Estado do Pará. Rev Soc Bras Med Trop. 2008;41(4):334-7.

15. Adán CBD, Diniz AR, Perlatto D, Hirai FE, Sato EH. Dez anos de doação de córneas no Banco de Olhos do Hospital São Paulo: perfil dos doadores de 1996 a 2005. Arq Bras Oftalmol. 2008;71(2):176-81.

16. Rech TH, Rodrigues Filho ÉM. Entrevista familiar e consentimento. Rev bras ter intensiva. 2007 jan-mar;19(1):85-9.

17. Chávez J, Campana S, Haas P. Panorama da hepatite B no Brasil e no Estado de Santa Catarina. Rev Panam Salud Publica. 2003;14(2):91-6.

18. Costa M. Hepatite B e hepatite C: estudo de incidência 1995-1997. Rev Port Saude Publica. 1999;17(2):47-54.

19. Silveira T, Fonseca J, Rivera L, Fay O, Tapia R, Santos J. Hepatitis B seroprevalence in Latin America. Pan Am J Public Health. 1999;6(6):378-83.

20. Focaccia R, Conceiçao, O JG, Junior, H S. Estimated prevalence of viral he patitis in the general population of the municipality of São Paulo, measured by a serologic survey of a stratified, randomized and residence-based population. Braz J Infect Dis. 2002;2:34-5.

21. Kaplan JE, Lhabbaz RF, Murphy EL, Hermansen S, Roberts C, Lar R. Male to female transmission of human T-cell lymphotropic virus types I and II: association with viral load. Acquir immune defic syndr human Retrovirol. 1996;Jun;12(1):193-201.

22. d'Orsi E. O perfil epidemiológico da AIDS. Florianopolis: Diretoria de Vigilância Epidemiológica, Secretaria de Estado da Saúde de Santa Catarina; 2006.

23. Pechansky F, Diemen L, Kessler F, Boni RD, Surrat H, Inciardi J. Preditores de soropositividade para HIV em indivíduos não abusadores de drogas que buscam centros de testagem e aconselhamento de POA, Rio Grande do Sul, Brasil. Cad Saúde Pública. 2005;21(1):266-74.

24. Kupek E. Transfusion risk for hepatitis B, hepatitis C and HIV in the state of Santa Catarina, Brazil, 1991-2001. Braz J Infect Dis. 2004 Jun;8(3):236-40.

25. Dias JCP, Schofield CJ. Controle da transmissão transfusional da doença de Chagas na Iniciativa do Cone Sul. Rev Soc Bras Med Trop. 2008;31(4):373-83.

26. Dias JCP. Notes about of Trypanosoma cruzi and yours bio-ecology characteristcs with agents of the transmission by meals. Rev Soc Bras Med Trop. 2006;39(4):370-5.

27. Chagas B, Pereira J. Seroprevalence of Chagas disease infection in the State of Piauí, 2002. Rev Soc Bras Med Trop. 2006;Nov-Dec;39(6):530-9. 
28. Ko W, Chu S, Lee Y, Lee P, Lee C, Chao S. Successful prevention of syphilis transmission from a multiple organ donor with serological evidence of syphilis. Transplant Proc. 1998;Nov;30(7):3667-8.

29. Riedner G, Rusizoka M, Todd J, Maboko L, Hoelscher M, Mmbando D, et al. Single-dose azithromycin versus penicillin $\mathrm{G}$ benzathine for the treatment of early syphilis. N Engl J Med. 2005;Sep;353(12):1236-44.

30. Debattista J, Dwyer J, Anderson R, Rowling D, Patten J, Mortlock M. Screening for syphilis among men who have sex with men in various clinical settings. Sex Trans Infect. 2004;80:505-8.

31. Vitek CR, Gracia FI, Giusti RA, Fukuda K, Green DB, Castillo LC. Evidence for sexual and mother to child transmission of human $\mathrm{T}$ lymphotropic virus type II among Guaymi Indians, Panama. J Infect Dis. 2001;Apr;171(4):1022-6.

32. Galvão BC, Loures L, Rodrigues L, Sereno A, Junior OF, Franco L. Distribution of human T-lymphotropic virus type I among blood donors; a nationwide Brazilian study. Transfusion. 1997;37:242-3.

33. Chen Y, Linc H, Chou P. A population - based epidemiological study of human T-cell leukemia virus type I infeccion in Kin - Hu, Kinmen. Int J Cancer. 1996;65:569-73.

34. Poulsen AG, Gallo D, Biggar RJ. Human T-cell leukaemia/lymphoma virus type I and type II in Guinea-Bissau, a portuguese speaking country of West Africa: risk factors and impact on survival. 8th International Conference on Human Retrovirology: HTLV. Rio de Janeiro, Brazil 1997. p. 9-13.
35. Biglione MPM, Crespo O, Severich I, Martinez PL, Libonatti O. High prevalence of human T-cell leukaemia/lymphoma virus infection in Argentinian blood donors: A new human T-cell leukaemia/lymphoma virus endemic area? J Acquir Immune Defic Syndr Hum Retrovirol. 1999;20:101-2.

36. Kealey GP, Aguiar J, Lewis RW, RG RGS, Bale-Jr JF. Cadaver skin allografts and transmission of human cytomegalovirus to burn patients. J Am Coll Surg. 1996;Mar;182(3):201-5.

37. Bale JJ, Kealey G, Massanari R. The epidemiology of cytomegalovirus infection among patients with burns. Infect Control Hosp Epidemiol1990;11(1):17-22.

38. Preiksaitis JK, Brown L, McKenzie M. The risk of cytomegalovirus infection in seronegative transfusion recipients not receiving exogenous immunosuppression. J Infect Dis. 1988;157(3):523-9.

39. Goodrich JM, Mori M, Gleaves CA. Early treatment with ganciclovir to prevent cytomegalovirus disease after allogeneic bone marrow transplantation. N Engl J Med. 1991;325(23):1601-7.

40. The Transplantaton Society. 3rd International Transplant Infectious Disease Conference; Praga 2007.

41. Kotton C. Zoonoses in solid-organ and hematopoietic stem cell transplant recipients. Clin Infect Dis. 2007;Mar;44(6):857-66. 